\title{
Outcome of Haemodialysis Arteriovenous Fistula in Baghdad, Iraq
}

\author{
Abdulsalam Y. Taha ${ }^{*}$, Omer Ahmed Diab ${ }^{2}$, Sabah N. Jaber ${ }^{2}$ \\ ${ }^{1}$ Department of Cardiothoracic and Vascular Surgery, School of Medicine, Sulaimania Teaching Hospital, Sulaymaniyah, Iraq; \\ ${ }^{2}$ Department of Cardiothoracic and Vascular Surgery, Baghdad Medical City Teaching Hospital, Baghdad, Iraq. \\ Email: *salamyt 1963@hotmail.com
}

Received November $8^{\text {th }}$, 2013; revised December $5^{\text {th }}$, 2013; accepted December $31^{\text {st }}$, 2013

Copyright (c) 2014 Abdulsalam Y. Taha et al. This is an open access article distributed under the Creative Commons Attribution License, which permits unrestricted use, distribution, and reproduction in any medium, provided the original work is properly cited. In accordance of the Creative Commons Attribution License all Copyrights (C) 2014 are reserved for SCIRP and the owner of the intellectual property Abdulsalam Y. Taha et al. All Copyright (C) 2014 are guarded by law and by SCIRP as a guardian.

\section{ABSTRACT}

Background: Vascular access (VA) is the life line for end stage renal disease (ESRD). Though there are many methods of VA, native arteriovenous fistula (AVF) is the oldest and the best. AVFs are prone to develop some complications. The aim of this combined prospective and retrospective clinical study was to assess the outcome of native haemodialysis AVF in Baghdad Medical City Teaching Hospital, Baghdad, Iraq. Materials and Methods: Sixty-seven patients (43 males and 24 females) with ESRD were studied over 6 months, from April 1 to September 30, 2013. Demographic and clinical features as well as co-morbidities were checked. Allen's test and examination of upper limb superficial veins were performed. Radio-cephalic or brachio-cephalic AVFs were created mostly under local anaesthesia using artery-side to vein-end anastamoses. Complications were noted during a follow-up period of 2 weeks to 6 months. Results: The mean age was $51.2 \pm 14.4$ years. Fistulas $(n=81)$ were mostly brachio-cephalic $(n=74,91.4 \%)$. One fifth of patients were diabetics and $58.2 \%$ were hypertensive. End of the vein to side of the artery was used in $\mathbf{9 2 . 5 \%}$. All fistulae functioned primarily. Significant complications were thrombosis $(n=18,22.2 \%)$, aneurysms $(n=3,4.5 \%)$ and steal syndrome $(n=3,13.6 \%)$. Distal oedema, venous congestion, wound infection and seroma were managed conservatively. Three surgical revisions were required, one for a large aneurysmal dilation (aneurysmectomy and vessel ligation) and two for an evacuation of seroma. Conclusion: AVF initial success was good. Late complications such as aneurysms and steal syndrome were almost within the reported rates whilst thrombosis was high.

\section{KEYWORDS}

\section{ESRD; Vascular Access; Native AVF; Complications}

\section{Introduction}

End stage renal disease has become a public health concern worldwide, with recent reports showing that the total number of ESRD patients has been growing dramatically [1]. The rising prevalence is due largely to two main factors: the ageing of the population and the global epidemic of diabetes. ESRD has a significant effect on the quality of life and life expectancy [1].

The ideal and proper treatment option of ESRD is renal transplantation. However, many patients do not have the opportunity to be treated as such or they need to wait

\footnotetext{
${ }^{*}$ Corresponding author.
}

for a variable period of time till they get this chance. Additionally, some patients develop rejection of the transplanted kidney. Such patients need to be managed by haemodialysis. Vascular access is vital for such patients. Though there are many methods of vascular access for haemodialysis (AVFs, arteriovenous grafts-AVG-and cuffed central venous catheters-CVCs) [2], native AVF is the oldest and the best. AVFs are prone to develop their own complications, which may result from surgical faults and/or an improper care in the dialysis units.

Historically, the native AVF was born in 1966 when Brescia et al. published their landmark account of 14 side-to-side anastamoses between the radial artery and 
cephalic vein at the wrist [3].

The aim of this study was to assess the outcome of native haemodialysis AVF in our institution in view of the current standards.

\section{Materials and Methods}

Sixty seven patients (43 males and 24 females) with ESRD were included in this study. Patients' informed consents and approval of the hospital Ethics Committee were obtained. There were 2 groups of patients; a prospective group $(n=45)$ referred to Vascular Surgery Unit, Baghdad Medical City Teaching Hospital over a 6 month period (April 1, 2013 to September 30, 2013) for creation of native AVF in the upper extremity and a retrospective group $(\mathrm{n}=22)$ of patients operated upon by the same surgeon(s) prior to April 12013 and seen in the Haemodialysis Unit. Other modes of vascular access like CVCs and AVGs were excluded. The patients were thoroughly assessed clinically noting the duration of the disease, co-morbidities such as DM, hypertension and peripheral arterial disease (PAD). Prior HD via CVC was recorded if present. Allen's test was performed to ensure patency of palmer arch and the superficial (particularly cephalic) veins were assessed looking for a good size vein. The patients were warned not to have venipunctures or intravenous cannulation while waiting for surgery. Oedema of upper limb and prominent collateral veins on shoulder and chest wall were observed when present as signs of central vein stenosis. The standard policy was to place the AVF at non-dominant wrist (radio-cephalic). Elderly patients, as well as patients with co-morbidities such as DM and PAD and those with non-available superficial vein at wrist were offered AVF at elbow (brachio-cephalic). Operations were done under local anaesthesia. Artery-side to vein-end anastomosis technique was used without a drain. Light dressing was applied. Function of AVF was judged by feeling a local thrill and listening to a bruit. Most operations were done by a senior registrar well trained in fistula surgery or occasionally by the senior surgeon. The patients were kept in the hospital up to 24 hours, after which discharged home if no complica- tions happened. A 10 day course of antibiotics was started one day before operation. Follow-up in the dialysis unit continued up to 6 months.

\section{Results}

Adult males constituted the majority as shown by Table 1.

Most of the operations were left-sided brachio-cephalic AVFs. This is evident in Table 2.

Table 3 displays the number of operations performed in this study. Most patients (82.1\%) had one AVF. It is worthy to observe that almost $90 \%$ of patients had HD via CVCs prior to AVF creation.

Early and late complications are shown in Table 4. Most of the early complications were managed conservatively. Significant complications included thrombosis, steal syndrome and aneurysms.

Table 5 displays details of thrombosis in this study. Most of the affected patients were females with brachiocephalic AVFs whereas most of the thromboses were late (>30 days).

Three patients developed arterial steal syndrome. The details are shown in Table 6.

Table 7 reveals characteristics of AVF aneurysms complicating three patients in this study.

Table 1. Age and sex distribution.

\begin{tabular}{cccc}
\hline Age (year) & Males, $\mathrm{n}$ & Females, $\mathrm{n}$ & Total, $\mathrm{n}(\%)$ \\
\hline $0-10$ & 1 & 0 & $1(1.5)$ \\
$11-20$ & 1 & 0 & $1(1.5)$ \\
$21-30$ & 1 & 2 & $3(4.5)$ \\
$31-40$ & 2 & 5 & $7(10.4)$ \\
$41-50$ & 14 & 8 & $22(32.8)$ \\
$51-60$ & 10 & 4 & $14(20.9)$ \\
$61-70$ & 11 & 3 & $14(20.9)$ \\
$71-80$ & 3 & 2 & $5(7.5)$ \\
Total & $43(64.2)$ & $24(35.8)$ & $67(100)$ \\
\hline
\end{tabular}

Table 2. Types vs. sides of AVFs.

\begin{tabular}{ccccc}
\hline Type & Right & Left & Bilateral & Total, $\mathrm{n}(\%)$ \\
\hline Radio-cephalic & 4 & 1 & 1 & $7(8.6)$ \\
Brachio-cephalic & 6 & 48 & 10 & $74(91.4)$ \\
Total & 10 & 49 & 11 & $81(100)$ \\
\hline
\end{tabular}

Table 3. Number of operations per patient.

\begin{tabular}{cccccc}
\hline Operation, $\mathrm{n}$ & 1 op. & 2 ops & 3 ops & 4 ops $^{*}$ & CVC \\
Patients, $n$ & $55(82.1)$ & $11(16.4)$ & 0 & 1 & $60(89.6)$ \\
\hline
\end{tabular}

\footnotetext{
"A lady of 39 a known case of SLE and ESRD for 26 years had had 4 thrombosed fistulas (2 radio-cephalic and 2 brachio-cephalic).
} 
Table 4. Complications.

\begin{tabular}{ccc}
\hline Complication & Patient, $\mathrm{n}(\%)$ & Comments \\
\hline Thrombosis & $14(20.9)$ & AVF thrombosis, 18 (22.2); late, $\mathrm{n}=15$ and early, $\mathrm{n}=3$ \\
AVF aneurysm & $3(4.5)$ & Details are mentioned in Table 7. \\
Arterial steal syndrome & $3(13.6)$ & Details are mentioned in Table 6. \\
Distal oedema & $25(37.3)^{*}$ & \\
Venous congestion & $8(11.9)^{*}$ & *All were managed conservatively. \\
Ooze & $4(6)^{*}$ & \\
Haematoma & $6(9)^{*}$ & 2 patients required evacuation \\
Wound infection & $1(1.5)^{*}$ & \\
Seroma & $8(11.9)$ &
\end{tabular}

Table 5. Details of cases of AVF thrombosis.

\begin{tabular}{cccccc}
\hline Group & Males & Females & AVF, $\mathrm{n}$ & Type & Onset \\
\hline Prospective & 3 & 7 & $11^{*}$ & Brachio-cephalic, $\mathrm{n}=16$ & Late, $\mathrm{n}=15$ \\
Retrospective & 1 & 3 & $7^{* *}$ & Radio-cephalic, $\mathrm{n}=2$ & Early, $\mathrm{n}=3$ \\
Total & 4 & 10 & 18 & 18 & 18 \\
\hline
\end{tabular}

*One female had 2 thrombosed fistulae; ${ }^{* *}$ One female had 4 thrombosed fistulae.

Table 6. Characteristics of patients with arterial steal syndrome.

\begin{tabular}{cccccc}
\hline Patient' Code & Age \& gender & Side, type \& age of AVF & Symptoms \& signs & CVL & Co-morbidities \\
\hline 49-R-13 & 71, M & R brachio-cephalic, 18 m & Pain at fingers during rest for 1 year & R IJV & DM \& VHC \\
57-R-13 & 71, F & L brachio-cephalic, $5 \mathrm{~m}$ & Pain at fingers during rest for 4 m & R IJV & HT \& VHC \\
66-R-13 & 64, M & L brachio-cephalic, $12 \mathrm{~m}$ & Rest pain at hand \& fingers for 12 m & R IJV & HT \&VHC \\
\multicolumn{2}{l}{ Mean age $=$ 68.7 yr } & Mean: $11.7 \mathrm{~m}$ & Mean $=9.3 \mathrm{~m}$ & & \\
\hline
\end{tabular}

\section{Discussion}

Clinical practice guidelines in both the US and Canada recommend the AVF as the preferred type of access for patients requiring chronic HD due to its longevity, low complication and low cost compared to AVGs and CVCs [4].

\subsection{Age and Gender}

The age of our patients ranged between 9 and 78 years with a mean of $51.2 \pm 14.4$. Most of them were young adults. The peak was in the fifth decade. This is similar to United Arab Emirates in which the highest incidence rate of ESRD was reported among the 45 - 55 years age group and to Kuwait in which the median of age among ESRD patients was 45 years [1]. In contrast, in Saudi Arabia, incidence and prevalence of ESRD were linked directly to increasing age [1]. Males constituted the majority (64.2\%). Hassanien et al. reviewed 44 articles describing the epidemiology of ESRD in countries of the Gulf Co-operation Council and found that the proportion of males was slightly higher than females [1]. Construction of haemodialysis AVF can be influenced by the age of the patient; the procedure may be difficult in the elderly (atherosclerosis) and in children (small vessels) [5]. It is often more difficult to construct a fistula in women than in men because their veins tend to be less well-developed [5].

\subsection{Co-Morbid Conditions}

Hypertension was reported in $58.2 \%$ of our patients whilst one fifth of them were diabetics. Diabetes has a direct effect on the success of AVF; as the distal arteries tend to be frequently diseased and unsuitable for creation of a successful AVF.

\subsection{Technical Aspects}

Local anaesthesia was used in all patients except two; a child of 9 and a man of 48 with a long standing brachio-cephalic AVF aneurysm to whom aneurysmectomy and ligation of vessels were done under GA. No drain 
Table 7. Characteristics of patients with aneurysms.

\begin{tabular}{l} 
Patients' summaries \\
\hline 02-P-13: 42 yrs, M, L brachio-cephalic AVF of 4 \\
months duration, very big pulsating swelling \\
at fistula site for 1 month, HT, \\
Exploration \& ligation of vessels. \\
62-R-13: 50 yrs, M, L brachio-cephalic \\
AVF of 6 months duration, pulsating swelling at \\
fistula site, HT \& VHC, managed by observation. \\
65-R-13: 44 yrs, M, L brachio-cephalic \\
swellings of cephalic vein, \\
HT \& VHB, managed by observation.
\end{tabular}

was used except in the abovementioned case. All fistulae functioned primarily with palpable thrill and audible bruit.

Eleven patients (16.4\%) had bilateral operations. This is most likely related to failure of one operation after a variable period of its creation or usage in dialysis which mandates creation of a new AVF in the same or opposite extremity.

The predominant left side of the operations could be due to the fact that most patients were right-handed. In addition, all CVCs in this study were placed in the right internal jugular vein; therefore, the right side should be avoided; creation of the fistula on the same side of CVC may result in failure of the fistula due to central venous stenosis complicating CVC placement. Initiation of HD with a CVC is associated with significantly higher mortality and morbidity including known risks such as infection, central vein stenosis, and thrombosis compared to individuals commencing HD with an AVF [4].

The predominant brachio-cephalic type in this series is most likely attributed to unavailability of veins at wrist region and the technical ease of creation of brachio-cephalic AVF. The radio-cephalic AVF is the golden choice and should have been used more frequently, though it is technically more demanding. Besides the small number of radio-cephalic AVFs ( $n=7,8.6 \%)$ performed in this study, most of them (4/7) were done on right side (this side should have been avoided unless the patients were left handed). There were no cases of trans- posed basilic vein AVF.

Creation of AVF for haemodialysis is technically demanding operation. It should not be left to a junior member of the surgical team unless he had gained the necessary skill. Most operations in this study $(n=49$, 73.1\%) were done by a skilled senior registrar, thus achieved a primary success.

The preferred anastomotic technique in AVF surgery today is the end of vein to artery side, although cutting of the vein end in an oblique angle may create functional problems due to stenosis [3]. This technique was the standard in this study; used in 62 cases (92.5\%).

Although intra-operative use of heparin is a standard routine followed by many authors to minimize thrombosis of the small vessels prior to clamping, it was used very infrequently $(n=4,6 \%)$ in this study. This was probably related to the well known bleeding tendency in ESRD patients which may be considered by the operating surgeon as a substitute to the use of heparin. Moreover, the fear from postoperative ooze and hematoma formation if heparin was used routinely may be another factor.

\subsection{Post-Operative Care}

The patients in this study were discharged home very early (within 1 to 24 hours). This is due to shortage of beds in our institution which cares for a huge number of trauma patients and the fact that most patients $(n=60)$ lived in Baghdad. However, we believe that a patient 
with recently created AVF for haemodialysis needs to be admitted a bit longer to allow for adequate patient monitoring and diagnosing potential complications such as bleeding and early thrombosis as well as to receive prophylactic antibiotics.

\subsection{Follow-Up}

Follow-up was good as each prospective patient was seen at least once by the operating surgeon with a mean of 3 visits whilst all retrospective patients except one were seen twice.

\subsection{AVF Thromboses}

AVF thromboses are the most frequent complications of $\mathrm{AV}$ access for haemodialysis [6]. The mean problem-free patency period after creation of native fistulas is approximately 3 years [7]. All observations and publications reported to date indicate that failure of native fistulas occurs most commonly as a result of narrowing of the outflow vein. Historically, native fistula malfunction and thrombosis were treated by using surgical thrombectomy and revision, resulting in the eventual exhaustion of the veins and the need to create a new access. Initially applied in the 1980s, percutaneous techniques such as percutaneous transluminal angioplasty (PTA), thrombolysis, and mechanical thrombectomy allowed the treatment of stenosis and fistula thrombosis without surgery [7]. In the present study, despite the high number of cases of AVF thromboses (22.2\%), duplex-ultrasound and fistulography were not used to evaluate the underlying causes and neither PTA nor surgery were used in the treatment of this complication. Probably some of these thrombosed fistulae could be saved if proper radiological and/or surgical interventions were used in the correct time.

\subsection{Arterial Steal Syndrome}

occurs in $5 \%-15 \%$ of cases when the brachial artery is used, or 10 times that of radio-cephalic AVFs. Treatment of steal includes observation of developing symptoms in mild cases. Balloon angioplasty is the appropriate intervention for an arterial stenosis [8]. In the present study, $3 / 22$ patients (13.6\%) developed arterial steal symptoms in the form of rest pain at hand and fingers (stage III). This incidence is within the previously reported 5\% - 15\% incidence [8,9]. All patients were elderly (mean age: 68.7 year), with co-morbidities (hypertension $n=2, D M n=1$ and VHC $\mathrm{n}=3$ ), all had brachio-cephalic AVFs for a mean duration of about 1 year.

\subsection{AVF Aneurysms}

One complication of haemodialysis AVF is that of aneurysmal dilatation, which can cause rupture and potentially fatal haemorrhage. The incidence of aneurysm forma- tion is approximately $5 \%$ to $6 \%$. Our incidence was slightly lower, 3/67 (4.5\%). It is useful, in AVF aneurysm, to do first a fistulogram as a proximal stenosis may be present, and in which case the patient first requires angioplasty. Previously described methods for treating this condition include ligation or resection of the AVF. This is effective and will eliminate the risk of bleeding but it is also wasteful because the otherwise functional AVF is sacrificed. These patients will require a temporary vascular access catheter insertion while a new AVF is created at another site [10]. Hong-Yee Lo and SeckGuan Tan described a new method in the treatment of this condition-plication. It involves plicating the excess free wall of the aneurysm with sutures and does not require resection or anastomosis [10]. Valentine and Aworanti from Jamaica in 2010 described an (effective) method for treatment of AVF aneurysms by excision of the aneurysmal sac and repair of the artery followed by creation of a new AVF at the same setting via the same incision. This method, as the authors believe, would save patients having to have a second surgical procedure for creation of a new AVF [11].

In this study we faced 2 cases of true aneurysms of outflow vein of AVF; both were managed by observation as their size was not too big to warranty intervention though none had a proper assessment by DUS or fistulography. The third case was a big pseudoaneurysm with impending rupture; therefore, it deserved exploration and ligation of vessels.

\subsection{Mortality}

There was no fistula-related death in this series. Two patients died 4 months after creation of fistula. In the study of Hassanein et al., the most common cause of death was cardiovascular disease and sepsis [1].

\subsection{There Are Several Shortcomings to This Study}

1) No patient had preoperative US examination of upper limb veins 2) Paradoxically, brachio-cephalic AVFs were used more frequently than radio-cephalic fistulas 3) No proper assessment of the complications by USG and fistulography and no radiological or surgical intervention performed to salvage fistulae complicated by aneurysms and arterial steal.

\section{Conclusion}

AVF initial success was good. Late complications such as aneurysms and steal syndrome were within the reported rates whilst thrombosis was high.

\section{REFERENCES}

[1] A. A. Hassanien, F. Al-Shaikh, E. P. Vamos, G. Yade- 
garfar and A. Majeed, "Epidemiology of End-Stage Renal Disease in the Countries of the Gulf Cooperation Council: A Systematic Review,” JRSM Short Reports, Vol. 3, No. 6, 2012, p. 38. http://shr.sagepub.com/content/3/6/38 http://dx.doi.org/10.1258/shorts.2012.011150

[2] K. Pantelias and E. Grapsa, "Vascular Access for Haemodialysis,” In: M. G. Penido, Ed., Technical Problems in Patients on Haemodialysis, InTech, 2011.

http://www.intechopen.com/books/technical-problems-in-p atients-on-hemodialysis/vascular-access-for-hemodialysis

[3] D. M. Hentschel, "Vascular Access for Hemodialysis," Nephrology Rounds, Vol. 6, No. 1, 2008, pp. 304-305.

[4] "CANNT Journal: The Culture of Vascular Access Cannulation among Nurses in a Chronic Haemodialysis Unit (Report) (monograph online)," Canadian Association of Nephrology Nurses \& Technologists, London, 2010. http://vodoojester.blogspot.com/2012/03/culture-of-vascu $\underline{\text { lar-access-cannulation.html }}$

[5] A. S. Y. Taha, "Provision of Vascular Access for Chronic Uremic Patients by Subcutaneous Arterio-Venous Fistula in the Upper Extremity,” Basra Journal of Surgery, Vol. 6, No. 1, 2000, pp. 28-31.

[6] V. Mickley, "Stenosis and Thrombosis in Haemodialysis
Fistulae and Grafts: The Surgeon's Point of View," Nephrology Dialysis Transplantation, Vol. 19, No. 2, 2004, pp. 309-311. http://dx.doi.org/10.1093/ndt/gfg504

[7] C. T Sofocleous, J. Cerveira and S. G. Cooper, "Dialysis Fistulas,” Kyung J Cho (Chief Editor), 2013. http://emedicine.medscape.com/article/419393-overview

[8] J. Malik, V. Tuka, Z. Kasalova, E. Chytilova, M. Slavikova, P. Clagett, et al., "Understanding the Dialysis Access Steal Syndrome. A Review of the Etiologies, Diagnosis, Prevention and Treatment Strategies," The Journal of Vascular Access, Vol. 9, No. 3, 2008, pp. 155166.

[9] V. Mickley, "Steal Syndrome-Strategies to Preserve Vascular Access and Extremity,” Nephrology Dialysis Transplantation, Vol. 23, No. 1, 2008, pp. 19-24. http://dx.doi.org/10.1093/ndt/gfm673

[10] H.-Y. Lo and S.-G. Tan, "Arteriovenous Fistula Aneurysm-Plicate, Not Ligate," Annals of the Academy of Medicine, Singapore, Vol. 36, No. 10, 2007, pp. 851-853.

[11] C. S. P. Valentine and O. Aworanti, "Surgical Management of Aneurysms of Arteriovenous Fistulae in Hemodialysis Patients: A Case Series,” Open Access Surgery, Vol. 3, 2010, pp. 9-12. 